\title{
Simple Sextant Calibration
}

\author{
D. W. Kerst
}

WHEN the scale readings of a sextant are suspected of error, or if an assurance of scale accuracy is needed, optical equipment for establishing angles is rarely available. Comparison with another sextant is possible, but if the arc between two stars were measured by bringing them into coincidence this could be compared with the calculated angular distance. To calculate the distance from a spherical triangle, knowing the stars' declinations and right ascensions, would be laborious and the refraction corrections would be difficult to apply-as they were in the old lunar distance methods. It is far simpler, in the northern hemisphere, to take Polaris as one of the two stars. If the other is within an hour of its meridian passage, so that the measured arc is approximately vertical, the usual corrections for refraction are sufficient. Also the calculated angular distance will then be simply $90^{\circ}-\delta$, where $\delta$ is the declination of the star selected. This calculated distance is therefore subject to two corrections :

(i) refraction corrections for both stars,

(ii) a correction for Polaris not being exactly at the pole.

If the two stars are on opposite sides of the zenith, both of the refraction corrections must be added to the measured angle; if both stars are on the same side of the zenith, the correction for the lower star must be added to the measured angle and the correction for the higher star subtracted. To find the refraction corrections it is sufficient to know the approximate altitude of Polaris (i.e. the latitude); the altitude of the other star is then approximately that of Polaris plus or minus the measured angle.

To correct for Polaris not being exactly at the pole we may imagine we are at a point on the Earth where the second star is at the zenith and then use the Polaris tables in the Nautical Almanac. Since we have assumed a position under the second star, we use $360^{\circ}-$ S.H.A. (star) instead of L.H.A. Aries in entering the table to find $a_{0}$; we substitute the star's declination for 'latitude' in entering the table to find $a_{1}$; finally $a_{2}$ is taken out for the correct month. The corrected angle, between the assumed zenith and Polaris, to be compared with the sextant reading is then $90^{\circ}-\delta+a_{0}+a_{1}+a_{2}-\mathrm{I}^{\circ}$.

The Polaris tables used in this way would only allow the calibration of sextant angles less than $90^{\circ}$, but the zero latitude value for $a_{1}$ can be used for arcs up to $120^{\circ}$ with an error of not more than $0 . I^{\prime}$. Since the tables extend only to Lat. $68^{\circ} \mathrm{N}$, it is not possible to use the method for arcs of less than $22^{\circ}$.

In making calibration observations it is essential to have the telescope parallel to the plane of the sextant and, if errors exceeding $0 \cdot I^{\prime}$ are not to be introduced, it is also necessary to view the two stars very near the centre line of the telescope field; within a quarter of a degree if the measured arc is near to $120^{\circ}$, within $0.4^{\circ}$ at $60^{\circ}$ and within $0.6^{\circ}$ at $30^{\circ}$. For a star telescope with a $6^{\circ}$ field this means reading within a vertical band only between $\frac{1}{10}$ and $\frac{1}{5}$ of the total field width. If the sextant has a micrometer drum this may also have a systematic error, which 
can be checked by readings on a horizontal graduated scale at a distance of 30 or $40 \mathrm{ft}$. If the drum scale is uniform, regular steps on the drum will correspond to regular steps on the scale.

\section{Reporting Near Misses at Sea}

\section{Nicolson}

I. INTRODUCTION. Not surprisingly much of the work that has been done on collision at sea has involved the study of collisions which have actually occurred. There must however be a wealth of untapped data relating to 'near misses' judging by the hair-raising stories often told by ships' officers. These accounts, sometimes of ships passing one another so closely in fog that human sounds have been heard, are doubtless subject to embellishment but are, nevertheless, the stuff that nightmares are made of.

An interesting point which (perhaps predictably) features in many such stories is that the navigator presents himself as a victim of circumstances rather than one who has suffered the effects of a wrong decision. Curiously the 'other ship' sometimes shares this diplomatic immunity. Discounting 'acts of God' then, is there room for a category of encounter in which blame is minimized or even ruled out? In the discussion following a recent paper ${ }^{1}$ Captain F. J. Wylie states that between 1948 and 1966 most of the collisions he had studied which had come to court had, according to the Judge, been due to personal error (generally related to the Rules or misinterpretation of radar information). This no doubt reflects the situation in a true light provided one accepts that it may occasionally be necessary to distinguish between 'personal error' and blame. The two might not go together, for example, when the error is due to overloading of the human system or under-exposure to congested waters-a view which appears to be gaining support in some quarters.

2. THE NEED FOR A REPORTING PROCEDURE. On the air side there is a well established system of reporting near misses and their circumstances ${ }^{2}$ but whilst many ships have for some time (and very effectively) carried a file of actual collision case studies ${ }^{3}$ no such system has yet been extended to selected near misses although useful work is being done in the Dover Strait. ${ }^{4}$

In the air, when one aircraft 'infringes the integrity' of another's airspace passing, say, within three miles, then a report is required. This procedure however is not of a punitive nature and the report goes, confidentially, before a joint airmiss working group who then categorize the incident according to its seriousness-'near hit', 'nominal infringement' \&c. Such a system, which runs parallel to but independently of the enquiry system, must reflect a need in shipping. The main difficulty with such an analogy, of course, lies in the vast difference in control factor between aircraft and shipping, and this being the case it is probably true to say that an appreciation of the build-up of events by the navigator would have more relevance to shipping than to aircraft. A marine reporting scheme would therefore be better directed at the training function than at research and the improvement of control systems, nevertheless adding to the statistical significance of existing research conclusions. 